\title{
Role of the neutron charge form factor in electron scattering from the three-nucleon system
}

\author{
R. A. Brandenburg and P. U. Sauer \\ Institut für Theoretische Physik, Technische Universität Hannover, 3000 Hannover, West Germany
}

(Received 24 March 1975)

\begin{abstract}
We investigate the effects which the experimental uncertainty of the neutron charge form factor can have on the charge form factors of ${ }^{3} \mathrm{H}$ and ${ }^{3} \mathrm{He}$.
\end{abstract}

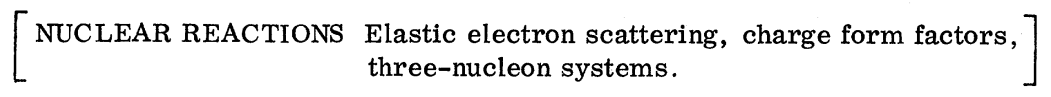

The uncertainty in the present knowledge of the neutron charge form factor has been shown by Bertozzi et al. ${ }^{1}$ to have noticeable effects in predicting electron scattering from heavy nuclei. In that reference, the charge form factor of the neutron is parametrized by the equation

$$
\begin{aligned}
G_{E}^{n}\left(q^{2}\right)= & {\left[1+\frac{1}{12} q^{2}\left(R_{\mathrm{av}}{ }^{2}-R_{\mathrm{o}}^{2}\right)\right]^{-2} } \\
& -\left[1+\frac{1}{12} q^{2}\left(R_{\mathrm{av}}{ }^{2}+R_{0}^{2}\right)\right]^{-2},
\end{aligned}
$$

with $R_{0}{ }^{2}=0.06 \mathrm{fm}^{2}$. $R_{\text {av }}$ equal to 0.63 and $1.07 \mathrm{fm}$ gives, respectively, the maximal and minimal form factors compatible with the experimental data, while $R_{\text {av }}$ equal to $0.80 \mathrm{fm}$ simulates the "standard fit" to the data.

We have calculated the charge form factors of the two possible three-nucleon bound states using

$$
F_{\mathrm{ch}}^{{ }^{3} \mathrm{H}\left({ }^{3} \mathrm{He}\right)}\left(q^{2}\right)=f_{3_{\mathrm{H}}}^{p(n)}\left(q^{2}\right) G_{E}^{p}\left(q^{2}\right)+f_{3_{\mathrm{H}}}^{n(p)}\left(q^{2}\right) G_{E}^{n}\left(q^{2}\right),
$$

where $G_{E}^{p(n)}$ represents the proton (neutron) charge form factors, while $f_{3 \mathrm{H}}^{p(n)}$ is the proton (neutron) body form factor of ${ }^{3} \mathrm{H}$. The body form factors are taken from a solution of the Faddeev equations ${ }^{3}$ using the ${ }^{1} S_{0}$ and ${ }^{3} S_{1}-{ }^{3} D_{1}$ partial waves of the Reid soft-core potential ${ }^{4}$ only. The body form factors, shown in Fig. 1, are related by

$$
f_{3_{\mathrm{H}}}^{p(n)}\left(q^{2}\right)=f_{3_{\mathrm{He}}}^{n(p)}\left(q^{2}\right) .
$$

For $G_{E}^{n}$ we use three parametrizations, i.e., the maximal and minimal functions of Eq. (1) and the "standard fit." The proton form factor $G_{E}^{p}$ is also taken from Ref. 2.

The effects of the experimental uncertainty in $G_{E}^{n}$ are smaller for ${ }^{3} \mathrm{He}$ than for ${ }^{3} \mathrm{H}$ since ${ }^{3} \mathrm{He}$ has only one neutron. The largest effects for both nuclei are found for momentum transfers in the region of the secondary maximum. Figure 2 shows the triton charge form factor in the region of its diffraction minimum and of its secondary maximum for the two limiting choices of $G_{E}^{n}$. Varying $G_{E}^{n}$ from its minimal to its maximal pa- rametrization moves the diffraction minimum towards smaller $q^{2}$ values by $0.4 \mathrm{fm}^{-2}$ and increases the secondary maximum by a factor of 1.5. In ${ }^{3} \mathrm{He}$ the diffraction minimum moves towards larger $q^{2}$ values by $0.2 \mathrm{fm}^{-2}$ while the secondary maximum increases by a factor of 1.1. Noting that both $G_{E}^{p}$ and $G_{E}^{n}$ are positive over the range of $q^{2}$ considered, the trends of these results can be understood qualitatively from Fig. 1 and Eq. (2). In the region of small momentum transfer, the percent effect decreases. In the range $6.0 \mathrm{fm}^{-2}$ $<q^{2}<12.0 \mathrm{fm}^{-2}$, the theoretical ${ }^{3} \mathrm{H}$ form factor is uncertain by approximately 10-15\% owing to the

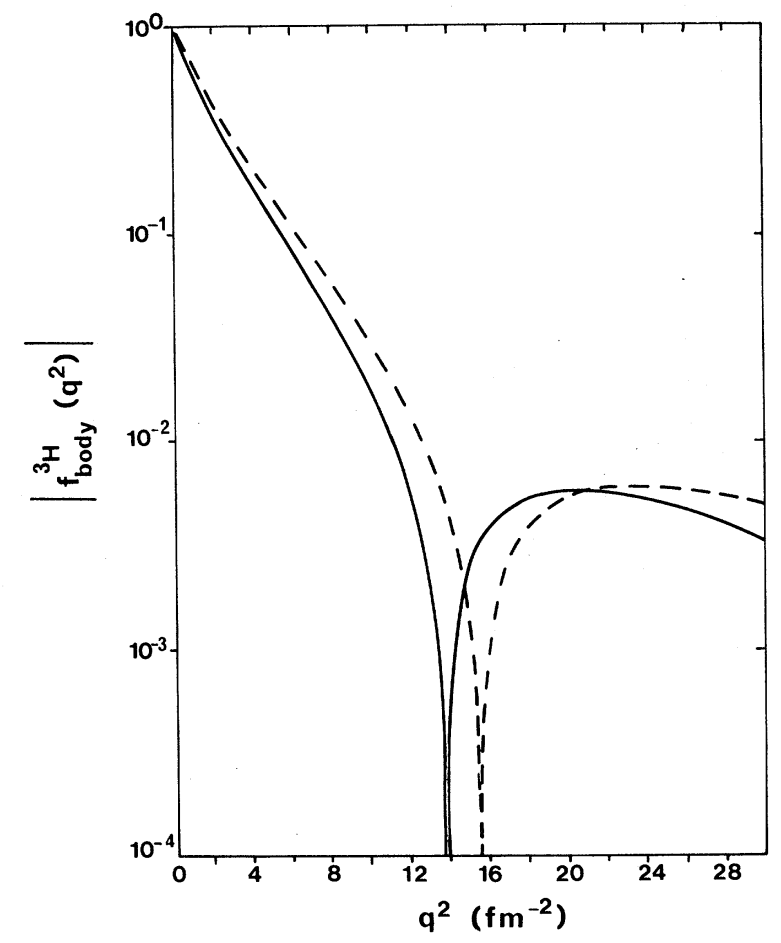

FIG. 1. The ${ }^{3} \mathrm{H}$ neutron and proton body form factors represented by the solid and dashed curves, respectively. 


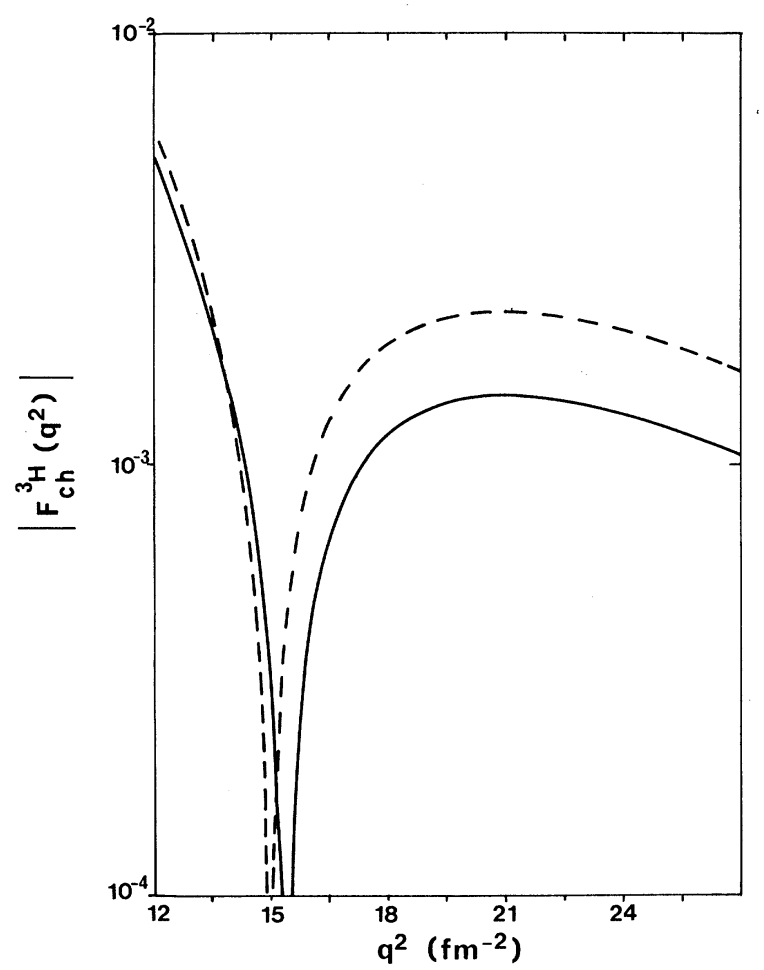

FIG. 2. The ${ }^{3} \mathrm{H}$ charge form factor using the minimal (solid curve) and maximal (dashed curve) parametrizations for $G_{E}^{n}$ according to Eq. (1).

uncertainty of $G_{E}^{n}$. For ${ }^{3} \mathrm{He}$, well outside the diffraction dip, the effect is between 4-9\%. Figure 3 illustrates the situation for ${ }^{3} \mathrm{He}$ and ${ }^{3} \mathrm{H}$. For each nucleus, the form factor calculated with the standard $G_{E}^{n}$ is plotted along with the difference between the form factors calculated using the maximal and minimal parametrizations of $G_{E}^{n}$. This difference represents the uncertainty of the theoretical form factors due to the experimental uncertainty of the neutron charge form factor.

The form factors which are presented here have the usual theoretical problems due to (i) the still incomplete knowledge of the two-nucleon potential; (ii) the technical inaccuracies in solving the three-

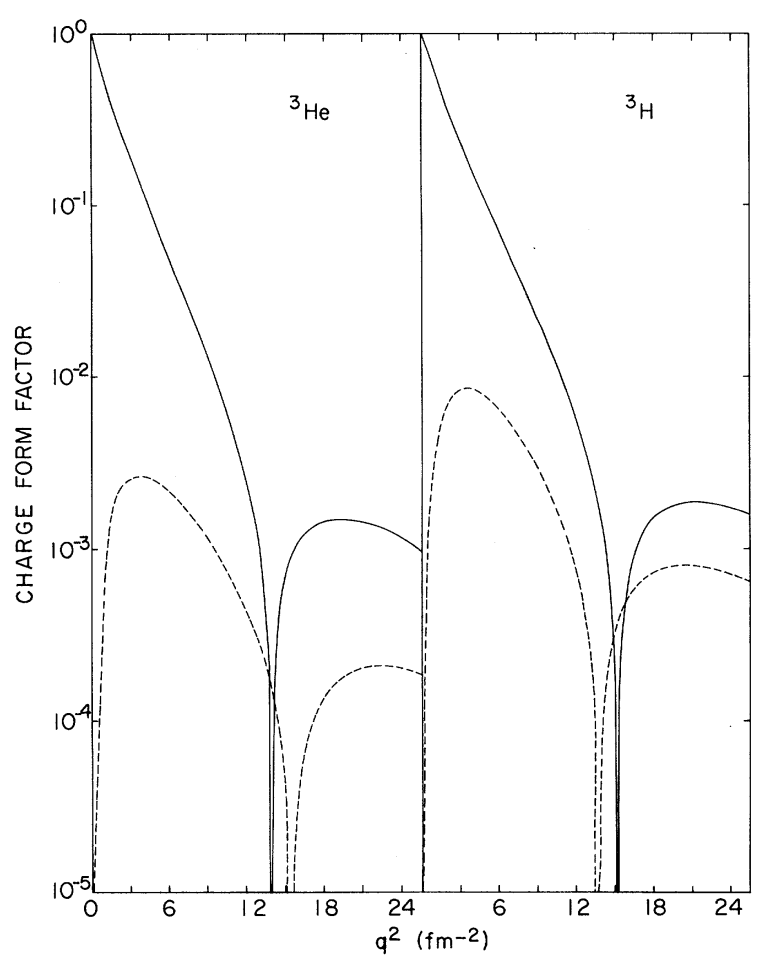

FIG. 3. Charge form factors for ${ }^{3} \mathrm{H}$ and ${ }^{3} \mathrm{He}$. The form factors with the standard $G_{E}^{n}$ of Ref. 2 (solid curve) are compared with the difference (dashed curve) between the form factors using the maximal and minimal parametrizations of $G_{E}^{n}$ according to Eq. (1). The absolute magnitudes are plotted.

nucleon Schrödinger equation, which seem to be greatest at high momentum transfers ${ }^{5}$; and (iii) the neglect of exchange-current effects whose size are still unclear. These theoretical uncertainties of the three-nucleon charge form factor may be even larger than those arising from the experimental uncertainty in the neutron form factor discussed in this note. However, even if the theoretical problems (i) to (iii) were removed, the effects of the experimental uncertainty in $G_{E}^{n}$ would persist and are expected to remain as sizable as shown in Figs. 2 and 3. This fact makes an accurate experimental determination of the neutron charge form factor important.
${ }^{1}$ W. Bertozzi, J. Friar, J. Heisenberg, and J. W. Negele, Phys. Lett. 41B, 408 (1972).

${ }^{2}$ T. Janssens, R. Hofstadter, E. B. Hughes, and M. R. Yearian, Phys. Rev. 142, 922 (1966).

${ }^{3}$ The solution used in this note is an improved calculation using the same techniques as E. P. Harper, Y. E.
Kim, and A. Tubis, Phys. Rev. Lett. 28, 1533 (1972). ${ }^{4}$ R. V. Reid, Ann. Phys. (N.Y.) 50, 411 (1968).

${ }^{5}$ R. A. Brandenburg, Y. E. Kim, and A. Tubis, Phys. Lett. 49B, 205 (1974); M. R. Strayer and P. U. Sauer, Nucl. Phys. A231, 1 (1974). 